\title{
Impact of depressive symptoms on subjective well-being: the importance of patient-reported outcomes in schizophrenia
}

This article was published in the following Dove Press journal:

Patient Preference and Adherence

26 September 2011

Number of times this article has been viewed

\author{
Jorge Mauriño' \\ Julio Sanjúan ${ }^{2}$ \\ Josep Maria Haro ${ }^{3}$ \\ Teresa Díez' \\ Javier Ballesteros ${ }^{4}$
}

'AstraZeneca Medical Department, Madrid, Spain; ${ }^{2}$ Department of Psychiatry, University of Valencia, CIBERSAM, Valencia, Spain;

${ }^{3}$ Parc Sanitari Sant Joan de Deu, CIBERSAM, Sant Boi de Llobregat, Spain; 'Department of NeurosciencePsychiatry, University of the Basque Country, UPV/EHU, CIBERSAM, Leioa, Spain
Correspondence: Jorge Mauriño Serrano Galvache, 56, Parque Norte, Edificio Roble, (28033) Madrid, Spain Tel +34 9l 30I 9738

$\mathrm{Fax}+34913019606$

Email jorgealejandro.maurino@ astrazeneca.com
Objective: The subjective experience of psychotic patients toward treatment is a key factor in medication adherence, quality of life, and clinical outcome. The aim of this study was to assess the subjective well-being in patients with schizophrenia and to examine its relationship with the presence and severity of depressive symptoms.

Methods: A multicenter, cross-sectional study was conducted with clinically stable outpatients diagnosed with schizophrenia. The Subjective Well-Being under Neuroleptic Scale - short version (SWN-K) and the Calgary Depression Scale for Schizophrenia (CDSS) were used to gather information on well-being and the presence and severity of depressive symptoms, respectively. Spearman's rank correlation was used to assess the associations between the SWN-K total score, its five subscales, and the CDSS total score. Discriminative validity was evaluated against that criterion by analysing the area under the curve (AUC).

Results: Ninety-seven patients were included in the study. Mean age was 35 years (standard deviation $=10$ ) and $72 \%$ were male. Both the total SWN-K scale and its five subscales correlated inversely and significantly with the CDSS total score $(P<0.0001)$. The highest correlation was observed for the total SWN-K (Spearman's rank order correlation [rho] $=-0.59$ ), being the other correlations: mental functioning $(-0.47)$, social integration $(-0.46)$, emotional regulation $(-0.51)$, physical functioning $(-0.48)$, and self-control ( -0.41$)$. A total of 33 patients $(34 \%)$ were classified as depressed. Total SWN-K showed the highest AUC when discriminating between depressive severity levels $(0.84)$, followed by emotional regulation $(0.80)$, social integration $(0.78)$, physical functioning and self-control (0.77), and mental functioning (0.73). Total SWN-K and its five subscales showed a significant linear trend against CDSS severity levels $(P<0.001)$.

Conclusion: The presence of moderate to severe depressive symptoms was relatively high, and correlated inversely with patients' subjective well-being. Routine assessment of patient-reported measures in patients with schizophrenia might reduce potential discrepancy between patient and physician assessment, increase therapeutic alliance, and improve outcome.

Keywords: schizophrenia, subjective well-being, patient-reported outcome, depressive symptoms

\section{Introduction}

Schizophrenia is a severe mental disease with devastating effects on the lives of patients and their caregivers. ${ }^{1}$

The systematic assessment of patient perspective may provide valuable information that could be missed when only clinical evaluation is relied on. In chronic conditions such as schizophrenia, there are advantages to using patient-reported measures to understand complex needs and improve alliances between patients and clinicians. ${ }^{2}$ 
Although since the 1960s there has been some interest in the subjective experience of patients with psychoses, until recently it has received little attention as a patient-reported outcome. ${ }^{3,4}$ In the last two decades, several psychometric initiatives have lead to the validation of several scales on subjective experience with antipsychotic drugs, including the Dysphoric Response Index, the Neuroleptic Dysphoria Scale, the Drug Attitude Inventory, the Rating of Medication Influences, the Subjective Well-Being under Neuroleptic Scale (SWN), the Attitudes Towards Neuroleptic Treatment, the Medication Adherence Rating Scale, and the Personal Evaluation of Transition in Treatment Scale. ${ }^{5-12}$ These scales differ not only in their application framework and administration form, but also in the type of questions framed to the problem of subjective experience. ${ }^{13}$ The SWN scale is a patient-reported outcome designed to assess patients' subjective experiences regardless of their mental state and medication stabilization. Contrary to scales that focus exclusively on medicationrelated outcome, the SWN also assesses subjective perception in other dimensions of quality of life. ${ }^{3}$

Depressive symptoms are common in patients with schizophrenia, ranging up to $65 \% .^{14,15}$ Their presence has been associated with worse clinical outcome, work impairment, a poorer quality of life, and higher rates of relapse and suicide. ${ }^{16}$

The aim of this study was to assess the subjective wellbeing in a sample of stable outpatients with schizophrenia and to determine its relationship with the presence and severity of depressive symptoms.

\section{Methods}

A non-interventional, cross-sectional study was conducted in 20 mental health centers throughout Spain. The study was approved by the institutional review board of the Fundació Sant Joan de Deu (Barcelona, Spain) (Clinical Trial Registry \#NCT00749970). Written informed consent was obtained from all patients. The criteria for patient recruitment were: 18-65 years of age; a diagnosis of schizophrenia, schizoaffective disorder, or schizophreniform disorder according to the Diagnostic and Statistical Manual of Mental Disorders: Fourth Edition; Text Revision; ${ }^{17}$ follow-up as an outpatient; clinically stable as judged by the investigators, considering stable those patients in whom a change in disease severity was not expected within 1 month after recruitment and who have not had any significant changes in antipsychotic medication for at least 3 months prior to the enrollment.

The Subjective Well-being under Neuroleptic - short version (SWN-K) is a self-rated scale consisting of 20 questions concerning physical and mental function, self-control, emotional regulation, and social integration. The total score ranges from 20 to 120 points, with higher scores indicating greater well-being. This scale has been reported with good psychometric qualities for its potential utility in both clinical practice and clinical trials with schizophrenic patients treated with antipsychotic drugs. ${ }^{3,18}$

The Calgary Depression Scale for Schizophrenia (CDSS) is a physician-rated measure specifically designed to assess depression in patients with schizophrenia. ${ }^{19}$ It is a nine-item questionnaire with a global score range from 0 to 27 points. It has been validated in independent studies and recommended as the gold standard for assessing depression in schizophrenia for clinical trials. ${ }^{20}$

Demographic and clinical characteristics were described using mean, standard deviation, and 95\% confidence interval (CI) for continuous measures. Spearman's rank correlation was used to assess associations between SWN-K total score, its five subscales, and CDSS total score. Following previous results from the Spanish version of the CDSS, a 4/5 cut-off point was chosen to dichotomize the clinical sample between mild and moderate to severe depressive symptoms. ${ }^{21}$ The discriminative validity of the SWN-K and its subscales was evaluated against that criterion by analyzing the area under the receiver operating characteristics curve (AUC). Finally, to evaluate a dose-effect linear trend, an ordinal three-group depression severity was created by using cut-off points at $3 / 4$ and $6 / 7$ for the CDSS total score. The appropriateness, or not, of a dose-effect linear trend was then evaluated by comparing nested models with a likelihood ratio test: the model including the CDSS information coded as a three-level factor and the model with the CDSS included as a variate.

\section{Results}

A total of 97 patients were included in the statistical analysis. The mean age was 35 years (standard deviation $[\mathrm{SD}]=10$ ), with males comprising $72 \%$ of the sample. Most patients had a diagnosis of schizophrenia $(n=77)$, followed by schizoaffective disorder $(\mathrm{n}=18)$, and schizophreniform disorder $(\mathrm{n}=2)$. Mean SWN-K total score was $83.5(\mathrm{SD}=14.0)$. Demographic and clinical characteristics of the sample are shown in Table 1.

Thirty-three patients $(34 \%, 95 \% \mathrm{CI}=24 \%-44 \%)$ were classified as depressed using the $4 / 5$ points CDSS cut-off criterion.

Both the SWN-K total score and its five subscales correlated inversely and significantly with the CDSS total score $(P<0.0001)$. The highest correlation was observed for the 
Table I Patient demographics and clinical characteristics

\begin{tabular}{ll}
\hline & $\mathbf{N}=\mathbf{9 7}$ \\
\hline Age (years) mean (SD) & $35.0(10.0)$ \\
Gender (male) (\%) & 72.0 \\
Living status (alone) (\%) & 11.5 \\
Marital status (single) (\%) & 92.8 \\
Age at onset (years), mean (SD) & $22.0(8.0)$ \\
Number of hospitalizations (median) (SD) & $3.0(3.7)$ \\
CGI-S score (mean) (SD) & $3.6(1.3)$ \\
PANSS total score (mean) (SD) & $65.0(21.4)$ \\
SWN-K total score (mean) (SD) & $83.5(14.0)$ \\
\hline
\end{tabular}

Abbreviations: CGI-S, Clinical Global Impression - Severity Scale; PANSS, Positive and Negative Syndrome Scale; SWN-K, Subjective Well-Being under Neuroleptic scale - short version.

total SWN-K (rho $=-0.59)$; the other correlations with the CDSS were as follows: mental functioning $(-0.47)$, social integration $(-0.46)$, emotional regulation $(-0.51)$, physical functioning $(-0.48)$, and self-control $(-0.41)$ (Table 2).

SWN-K total score showed the highest AUC when discriminating among depressive severity levels (0.84), followed by emotional regulation (0.80), social integration (0.78), physical functioning, self-control (0.77), and mental functioning (0.73). Both the total SWN-K and its five subscales showed a significant linear trend against CDSS severity levels $(P<0.001)$ (Table 3).

\section{Discussion}

Although patients with schizophrenia may traditionally have been treated as passive recipients of treatment, in the last few decades they have increasingly been seen as active partners in care whose views and opinions matter. ${ }^{2} \mathrm{New}$ constructs and measures are shifting the emphasis from symptom management to maximizing the patient's quality of life and functional recovery. ${ }^{22}$ In fact, the US Food and Drug Administration has issued guidance for the use of patient assessments as effectiveness endpoints in clinical trials. ${ }^{23}$

Table 2 Spearman correlations and area under the curve between SWN-K and CDSS

\begin{tabular}{lll}
\hline SWN-K & $\begin{array}{l}\text { CDSS total score } \\
\text { rho }(95 \% \mathrm{CI}) *\end{array}$ & $\begin{array}{l}\text { CDSS 4/5 cut-off } \\
\text { AUC }(95 \% \mathrm{CI})\end{array}$ \\
\hline Total score & $-0.59(-0.7 \mathrm{I}$ to -0.44$)$ & $0.84(0.75$ to 0.92$)$ \\
Mental functioning & $-0.47(-0.6 \mathrm{I}$ to -0.30$)$ & $0.73(0.6 \mathrm{I}$ to 0.84$)$ \\
Social integration & $-0.46(-0.60$ to -0.29$)$ & $0.78(0.68$ to 0.88$)$ \\
Emotional regulation & $-0.5 \mathrm{I}(-0.64$ to -0.34$)$ & $0.80(0.70$ to 0.89$)$ \\
Physical functioning & $-0.48(-0.62$ to $-0.3 \mathrm{I})$ & $0.77(0.67$ to 0.87$)$ \\
Self-control & $-0.4 \mathrm{I}(-0.56$ to -0.22$)$ & $0.77(0.68$ to 0.87$)$ \\
\hline
\end{tabular}

Note: *All P-values $<0.000$ I.

Abbreviations: SWN-K, Subjective Well-being under Neuroleptic Scale - short version; CDSS, Calgary Depression Scale for Schizophrenia; AUC, area under the curve; $\mathrm{Cl}$, confidence interval.
Table 3 Discriminative performance of the SWN-K against CDSS depression levels

\begin{tabular}{|c|c|c|c|}
\hline SWN-K & $\begin{array}{l}\text { CDSS } \leq 3 \\
\text { Mean (SD) }\end{array}$ & $\begin{array}{l}4 \geq \text { CDSS } \leq 6 \\
\text { Mean (SD) }\end{array}$ & $\begin{array}{l}\text { CDSS } \geq 7 \\
\text { Mean (SD) }\end{array}$ \\
\hline Total score & $89.8(10.6)$ & $8 \mathrm{II}(10.0)$ & $66.9(12.7)$ \\
\hline Mental functioning & $17.0(3.0)$ & $15.8(3.4)$ & II.7 (4.2) \\
\hline Social integration & $17.8(3.3)$ & $16.3(3.3)$ & |3.2 (2.9) \\
\hline Emotional regulation & $18.9(2.9)$ & $15.8(2.7)$ & I4.0 (4.3) \\
\hline Physical functioning & $18.6(3.1)$ & $17.0(3.3)$ & I3.8 (3.3) \\
\hline Self-control & $17.6(2.9)$ & I $6.7(2.5)$ & $13.6(2.7)$ \\
\hline
\end{tabular}

Note: All P-values for linear trend $<0.001$.

Abbreviations: SWN-K, Subjective Well-being under Neuroleptic scale - short version; CDSS, Calgary Depression Scale for Schizophrenia; SD, standard deviation.

The subjective experience of psychotic patients toward treatment is a key factor in medication adherence, quality of life, and clinical outcome. ${ }^{3}$ Both the SWN ${ }^{9}$ and its 20 -item short version ${ }^{3}$ were developed to assess the subjective wellbeing of patients treated with antipsychotic drugs without focusing differently on adverse effects of treatment or symptoms severity. ${ }^{3,9}$ As a consequence, the SWN contents should overlap those assessed by independent clinician-rated outcomes regarding adverse effects and symptoms severity, as well as contents covered by other patient-reported measures concerning attitudes to medication. However, a recent publication does point to the practical independence of the SWN-K from the Drug Attitude Inventory, suggesting that attitudes to medication and well-being might represent nonoverlapping dimensions. ${ }^{18}$

Depression is a common symptom over the course of schizophrenia. ${ }^{14}$ Patients with depressive symptoms suffer significantly more negative symptoms at discharge, have impaired subjective well-being and functioning, and a higher risk of suicide. ${ }^{16,24}$ The high prevalence of depressive symptoms in schizophrenia and, in particular, its negative impact on prognosis make detection and treatment essential.

A limitation of this study was the lack of assessment of additional factors related to quality of life and patient well-being, such as comorbidity, substance abuse, type of antipsychotic treatment, or medication adherence.

The presence of moderate to severe depressive symptoms in the sample of clinically stable outpatients in this study was relatively high (34\%) and also correlated inversely with their subjective well-being. This highlights the relevance of recognizing depressive symptoms in patients with schizophrenia and the need for close monitoring.

Involving patients with schizophrenia in their own treatment is feasible, except perhaps in a few cases where 
individuals have severe cognitive dysfunction or extremely reduced insight. ${ }^{25}$

Therefore, systematic routine assessment, using both clinician-rated and patient-reported measures, might help to gather more in-depth clinical information and increase the patient's confidence.

\section{Disclosure}

This study was sponsored by AstraZeneca Spain. JM and TD are employees of AstraZeneca.

\section{References}

1. McGrath J, Saha S, Chant D, Welham J. Schizophrenia: a concise overview of incidence, prevalence, and mortality. Epidemiol Rev. 2008;30:67-76.

2. McCabe R, Saidi M, Priebe S. Patient-reported outcomes in schizophrenia. Br J Psychiatry Suppl. 2007;191(50):S21-S28.

3. Naber D, Moritz S, Lambert M, et al. Improvement of schizophrenic patients' subjective well-being under atypical antipsychotic drugs. Schizophr Res. 2001;50:79-88.

4. Marder S. Subjective experiences on antipsychotic medications: synthesis and conclusions. Acta Psychiatr Scand Suppl. 2005;427:43-46.

5. Singh MM, Kay SR. Dysphoric response to neuroleptic treatment in schizophrenia: its relationship to autonomic arousal and prognosis. Biol Psychiatry. 1979;14:277-294.

6. Hogan TP, Awad AG, Eastwood R. A self-report scale predictive of drug compliance in schizophrenics: reliability and discriminative validity. Psychol Med. 1983;13:177-183.

7. Awad AG. Subjective response to neuroleptics in schizophrenia. Schizophr Bull. 1993;19:609-618.

8. Weiden P, Rapkin B, Mott T, et al. Rating of medication influences (ROMI) scale in schizophrenia. Schizophr Bull. 1994;20:297-310.

9. Naber D. A self-rating to measure subjective effects of neuroleptic drugs, relationships to objective psychopathology, quality of life, compliance and other clinical variables. Int Clin Psychopharmacol. 1995;10 Suppl 3:133-138.

10. Kampman O, Lehtinen K, Lassila V, Leinonen E, Poutanen O, Koivisto A. Attitudes towards neuroleptic treatment: reliability and validity of the attitudes towards neuroleptic treatment (ANT) questionnaire. Schizophr Res. 2000;45:223-234.

11. Thompson K, Kulkarni J, Sergejew AA. Reliability and validity of a new Medication Adherence Rating Scale (MARS) for the psychoses. Schizophr Res. 2000;42:241-247.
12. Voruganti L, Awad AG. Personal evaluation of transitions in treatment (PETiT): a scale to measure subjective aspects of antipsychotic drug therapy in schizophrenia. Schizophr Res. 2002;56:37-46.

13. Barberá M, Sanjuán J, Munárriz M, Novella E, Santiago C, Simó M. Subjective experience with antipsychotics:quantitative evaluation. Actas Esp Psiquiatr. 2006;34:287-294. Spanish.

14. Siris SG. Depression in schizophrenia: perspective in the era of "Atypical" antipsychotic agents. Am J Psychiatry. 2000;157:1379-1389.

15. Majadas S, Olivares J, Galan J, Diez T. Prevalence of depression and its relationship with other clinical characteristics in a sample of patients with stable schizophrenia. Compr Psychiatry. 2011; Epub May 27, 2011.

16. Schennach-Wolff R, Obermeier M, Seemüller F, et al. Evaluating depressive symptoms and their impact on outcome in schizophrenia applying the Calgary Depression Scale. Acta Psychiatr Scand. 2011; 123:228-238.

17. American Psychiatric Association. Diagnostic and Statistical Manual of Mental Disorders: Fourth Edition; Text Revision. Washington, DC: American Psychiatric Association, 2000.

18. Sanjúan J, Haro JM, Mauriño J, Díez T, Ballesteros J. Validation of the Spanish version of the Subjective Well-being under Neuroleptic Scale (SWN-K) in patients with schizophrenia. Med Clin (Barc). 2011; Epub May 13, 2011.

19. Addington D, Addington J, Maticka-Tyndale E, Joyce J. Reliability and validity of a depression rating scale for schizophrenics. Schizophr Res. 1992;6:201-208.

20. Kontaxakis VP, Havaki-Kontaxaki BJ, Stamouli SS, Margariti MM, Collias CT, Christodoulou GN. Comparison of four scales measuring depression in schizophrenic inpatients. Eur Psychiatry. 2000;15: 274-277.

21. Sarró S, Dueñas RM, Ramírez N, et al. Cross-cultural adaptation and validation of the Spanish version of the Calgary Depression Scale for Schizophrenia. Schizophr Res. 2004;68:349-356.

22. Burns T. Evolution of outcome measures in schizophrenia. $\mathrm{Br}$ J Psychiatry Suppl. 2007;191:S1-S6.

23. US Department of Health and Human Services FDA Center for Drug Evaluation and Research, US Department of Health and Human Services FDA Center for Biologics Evaluation and Research, US Department of Health and Human Services FDA Center for Devices and Radiological Health. Guidance for industry: patient-reported outcome measures: use in medical product development to support labeling claims: draft guidance. Health Qual Life Outcomes. 2006;4:79.

24. Kim JH, Ann JH, Kim MJ. Relationship between improvements of subjective well-being and depressive symptoms during acute treatment of schizophrenia with atypical antipsychotics. J Clin Pharm Ther. 2011;36:172-178.

25. Hunter R, Cameron R, Norrie J. Using patient-reported outcomes in schizophrenia: The Scottish schizophrenia outcomes study. Psychiatr Serv. 2009;60:240-245.
Patient Preference and Adherence

\section{Publish your work in this journal}

Patient Preference and Adherence is an international, peer-reviewed, open access journal focusing on the growing importance of patient preference and adherence throughout the therapeutic continuum. Patient satisfaction, acceptability, quality of life, compliance, persistence and their role in developing new therapeutic modalities and compounds to

\section{Dovepress}

optimize clinical outcomes for existing disease states are major areas of interest. This journal has been accepted for indexing on PubMed Central. The manuscript management system is completely online and includes a very quick and fair peer-review system. Visit http://www.dovepress.com/ testimonials.php to read real quotes from published authors. 